\title{
Lightweight Games User Research for Indies and Non-Profit Organizations
}

\author{
Lennart E. Nacke \\ HCI Games Group \\ University of Waterloo, \\ Waterloo, Ontario, Canada \\ lennart.nacke@acm.org \\ Christiane Moser \\ Center for $\mathrm{HCI}$ \\ University of Salzburg, Austria \\ christiane.moser@sbg.ac.at
}

\section{Anders Drachen}

Aalborg University

Copenhagen, Denmark

anders.drachen@gmail.com

\author{
Pejman Mirza-Babaei \\ University of Ontario Institute of \\ Technology \\ Oshawa, Ontario, Canada \\ pejman@uoit.ca

\section{Andrea Abney} \\ Scopely \\ Los Angeles, CA, USA \\ andrea.abney@gmail.com
}

\section{Zhu (Cole) Zhenyu}

Interactive Entertainment Group

Tencent, Shenzhen, Guangdong,

China

zhenyuzhu@tencent.com

Permission to make digital or hard copies of part or all of this work for personal or classroom use is granted without fee provided that copies are not made or distributed for profit or commercial advantage and that copies bear this notice and the full citation on the first page. Copyrights for third-party components of this work the first pane. Copyright is held by the owner/author(s).

CHI'16 Extended Abstracts, May 07-12, 2016, San Jose, CA, USA

(c) Lennart Nacke, 2016. This is the author's version of the work. It is postedhere for your personal use. Not for redistribution. The definitive version was published in CHI EA '16 Proceedings of the 2016 CHI Conference Extended Abstracts on Human Factors in Computing Systems, https://doi.org/10.1145/2851581.2856504 http://dx.doi.org/10.1145/2851581.2856504

\begin{abstract}
The Games User Research (GUR) community has thrived at $\mathrm{CHI}$ with four workshops and a course since CHI 2012; all of these were well attended. In line with the \#chi4good spirit this year, the GUR field must advance towards demographics that will benefit from GUR but are currently underrepresented in the community: Small, independent developers, non-profit organizations, and academics that create mobile games, games for health or change, or educational games. This workshop will be a think tank for participants to construct collective knowledge, share and discuss. We plan to discuss topics online beyond the workshop via the International Game Developer Associations Special Interest Group on GUR, which serves as a basis for disseminating workshop outcomes and further discussion.
\end{abstract}

\section{Author Keywords}

Games User Research; Games 4 Health; Games for Change; User Experience; Usability; Playability; Games Research.

\section{ACM Classification Keywords}

K.8.0 PERSONAL COMPUTING; General - Games.

\section{Background}

Game User Research (GUR) is a research area focused on the development of techniques and tools to measure 


\section{Workshop Goals}

1. Discuss, design and develop lightweight GUR strategies and tools for situations where resources are minimal and cost: benefit ratio must be kept low.

2. Develop strategies for communicating lightweight GUR results to a broad range of stakeholders

3. Disseminate knowledge about lightweight GUR to the indie and other small-scale companies, as well as non-profit organizations, about how to take advantage of GUR players' behaviours and experiences to help game developers to improve on usability and player's experiences of their titles. Therefore, tools are used mostly for the purpose of evaluation and gaining insights from players to enhance game designs.

Video games are more popular, pervasive, and ubiquitous than ever. Everyone plays them: children and older adults alike. They are also developed by a more diverse set of people than ever. More than 250,000 games are found on the AppStore alone, and according to McGonigal [4] more than 3 billion hours are spent globally playing games every week. According to the Entertainment Software Association, in the US alone, there are 155 million active players, across all devices, and $42 \%$ play more than 3 hours per week. Roughly an equal amount of males and females play games, and the average age of people playing games is 35 years [2]. The games market has a projected total size of 100 billion by 2017 [7]. For both mobile and console, the top games generate the vast majority of the revenue, with top games generating incredible revenues, and a very long tail of titles with minimal earnings [8, 12].

Despite the immediate importance of user research in games (which succeed or fail on the quality of the user experience provided), GUR is not mature throughout the industry and associated academic and non-profit domains. Indie (i.e., small commercial) developers, non-profit organizations, and other small development units that have an interest in game development or gamification, often lack resources, knowledge, and experience to include GUR in the design and development process of their games. They also lack the necessary knowledge to communicate the results of GUR work to the various stakeholders involved in game de- velopment (e.g., programmers, designers, producers, or marketing), whom all benefit from GUR work but require different kinds of input. This miscommunication often results in bad player experiences, bugs, or flaws, which means that these games cannot compete with games developed with full GUR support.

Small game productions are also commonly resource starved, which means that hard choices have to be made on what to focus. Giving the lack of GUR

knowledge, this aspect of game development is often ignored or delayed until making changes becomes too expensive. In essence, a lack of GUR impacts sales, increases fail rates, and leads to a low persistence on the market [3]. Notably, games developed for educational or serious purposes often fail to reach a wide audience and thus real impact. There is thus a substantial need to support particularly these types of games that especially have purposes for good (e.g., education and training). The small developers are also a community that traditionally is not strongly represented at $\mathrm{CHI}$, and this workshop seeks to change that, by inviting these audiences directly, and by setting up a dissemination platform for the results generated at the workshop. The broader region where $\mathrm{CHI}$ 2016 is situated further supports this aim by having unique entrepreneurship environments.

We made significant progress on GUR methods in previous workshops (at CHI 2012 [9], CHI 2013 [5], CHI 2014 [13], CHI PLAY 2014 [1], CHI 2015 [11], CHI PLAY 2015 [10], each with more than 30 participants). However, we see strong value in this GUR workshop addressing the underrepresented small independent developers, non-profit organizations, and academics 
that create mobile games, games for health or change, or educational games in the GUR community.

Within the scope of this year's CHI, we are proposing to build a workshop targeting the "long tail" in game development (i.e., the marginal group outside the big studios, such as indie developers and non-profit organizations). Our goal is to understand, investigate, promote, and provide the value of GUR for these small companies and initiatives. We are interested in finding out, how they can benefit from existing GUR strategies, tools, and techniques, or how these need to be modified, adapted, or re-thought to meet their needs. It is necessary to discuss resource costs, training needs, value-to-cost ratios, as well as automation and remote testing capacities. The outcome of this workshop will be a collection of DIY (Do-It-Yourself) best practices, guidelines, tools, and techniques for this target group that is simple, lightweight, and cheap to apply.

\section{Media Strategy}

The organizers will be using the past CHI GUR workshops website to document the workshop's content. Participants will be encouraged to contribute to the GUR website with pre- and post-workshop tasks. For example, participants provide their biographies, position papers/presentation slides, questions (preworkshop) and the organizers summarize the workshop outcome (post-workshop). In this way, we hope to maximize the reach of the workshop. The workshop will promote the conference through some established online venues in the GUR domain (e.g., the IGDA GUR SIG, see below) and Game Research email lists. These sites will play a crucial role in publicizing the workshop and act as bases for pre and post workshop activities.
Some related social networks such as Twitter, Facebook, and LinkedIn will be used from these sites.

We are planning to extend the website from our past workshops to include the CfP and all necessary details and background information. The website can be found here:

$\rightarrow$ http://gur.hcigames.com/chi2016

After the workshop, the website will be used to archive the papers, talks (slides) from workshop participants, and the workshop outcome.

\section{Workshop Organization}

Pre-Workshop Plans

The workshop organizers commit to publicizing and promoting the workshop. We will distribute the call for participation on mailing lists, the \#chigur Twitter hashtag from last year's workshop to encourage community involvement and online social networking between workshop participants and the existing IGDA GUR SIG community. ${ }^{1}$

Before starting this workshop, we will ask the participants to prepare position papers, practice videos, or case study presentation slides, which will be posted on the website before the workshop. We also plan to run several surveys to gather some relevant information regarding the needs of non-profit organizations and independent developers. These insights will serve as a starting point for the roundtable discussions.

\footnotetext{
1 https://www.linkedin.com/grp/home?gid $=1873014$
} 


\section{Workshop Schedule \\ 09:00 - 09:15 Introduction}

09:15 - 09:25 Opening talk by organizers

09:25 - 11:30 Participants presentations

\section{1:30 Coffee Break}

12:00 - 13:00 Critique and feedback of the presented work

13:00 Lunch (Networking)

14:30 - 16:00 Round table discussion with survey insights

16:00 Coffee Break

16:30 - 17:30 Brainstorm for DIY tools

17:30 - 18:00 Closing Discussion; wrap up

19:30 Dinner (Networking)
Our materials will become available before the workshop start date on the dedicated website. This serves two purposes: (a) To prepare and facilitate the discussion during the workshop as attendees will be asked to read the works in advance and comment on them; and (b) to reach, stimulate, and share the workshop material with a wider audience.

\section{Workshop Structure}

The workshop will be run over a single day. During the initial orientation, workshop organizers and participants will present an overview of their work, the issues they face, and current best practices in their field. The afternoon sessions will provide an opportunity for participants to gather together into smaller teams and have a roundtable discussion. The workshop will end with a collection of guidelines and best practices to integrate GUR in the development cycle for low-budget projects.

\section{Post-Workshop Plans}

Workshop organizers will provide written summaries of the different activities from the workshop on the dedicated website, as well as adding digital copies of the group productions (i.e., poster and interactive whiteboard). The intention of the outcomes is to facilitate on-going dialogue between attendees and peers in the field. The results of the workshop will be presented at CHI 2016 in the form of a poster describing the workshop and a summary of findings.

\section{Workshop Outcomes}

Following the workshop, we aim to be able to pass on to the broader community a review of the event's talks and presentations, to promote on-going dialogue between workshop participants and other peers in the field. The workshop will facilitate community building by keeping a record of the participants' initial position papers, the snapshot of how players are addressed in current practice, and final roadmap identifying novel areas for research collaboration. This archive will be made available to the wider community by being offered to channels such as poster/video presentation at $\mathrm{CHI}^{\prime} 16$, the workshop's website, the CHI Games Community Facebook page, and Games Research Network email list.

\section{Call for Participation (CfP)}

The Games User Research (GUR) community has thrived at $\mathrm{CHI}$ with four workshops and a course since CHI 2012; all of these were well attended. In line with the \#chi4good spirit, the GUR field must advance towards demographics that will benefit from GUR, but are currently underrepresented in the community, such as small, independent developers, non-profit organizations and academics that create mobile games, games for health or change, and educational games. This workshop will be a think tank for everyone to construct collective knowledge, share and discuss among GUR professionals, non-profits, indie developers, and the interdisciplinary academics interested in the field.

This workshop is the first-of-its-kind GUR workshop that focuses on lightweight (guerilla) methods for GUR, e.g., a low-cost adaptation of eye-tracking methods to evaluate games user interface. Ultimately, the workshop serves as a springboard towards building a community of user researchers around the common issues and approaches when dealing with low budgeted projects.

Participants are invited to submit a 4-page position paper ( $\mathrm{CHI}$ extended abstracts format) or an abstract 
and slides/video (only recommended for industry participants). Content should highlight practical challenges related to applying GUR for indie or non-profit organizations.

Participants are asked to submit their proposals via EasyChair by 14 December for early acceptance or 13 January 2016 for final acceptance. A short biography of each author attending the workshop needs to be included (100-150 words). Submissions will be peerreviewed, and the organizing committee will select up to 20 participants according to relevance, quality of results, and research diversity. If accepted, at least one author must register for the workshop and one or more days of the $\mathrm{CHI}$ conference. Papers will also be compiled on the workshop website.

\section{Workshop's CfP released:}

30 November

Submissions deadline round 1:

18 December 2015

Early acceptance round for participants:

21 December 2015.

Submissions deadline round 2:

13 January 2016

Final acceptance round:

20 January 2016

Participants' final submissions:

12 February 2016.

\section{Workshop:}

May 7 or 8, 2016

\section{Workshop Organizers}

Lennart E. Nacke is an Associate Professor for Human-Computer Interaction and Game Design in the Faculty of Arts at the University of Waterloo. He is re- searching the cognitive and emotional side of playing games and affiliated with the Games Institute. Lennart has organized many GUR workshops and a course for $\mathrm{CHI}$ over the past five years; he also chaired the $\mathrm{CHI}$ PLAY 2014 and Gamification 2013 conferences and is currently the chair of the CHI PLAY steering committee. He has served on the steering committee of the IGDA GUR Special Interest Group (SIG) in the past. He is also currently editing a GUR book together with Pejman and Anders (see: http://www.gurbook.com).

Christiane Moser is a postdoctoral research fellow at the Center for HCI, University of Salzburg. Her recently finished PhD was on the topic of child-centered game development. She is currently involved in national and international research projects. She has much experience in running diverse user studies from the requirements analysis to evaluation of systems and games. Her interest is the iterative development and improvement together with users and players. She has already organized workshops at IDC 2011, MobileHCI 2011, FnG 2012, CHI 2013, ACE 2013, and CHI Play 2014. She chaired the Children Creativity Lab at ACE 2014.

Anders Drachen is a veteran Data Scientist and Associate Professor at Aalborg University (Denmark), as well as an analytics consultant. His work in the game industry as well as in data and game science is focused on game analytics, business intelligence for games, game data mining, game user experience, industry economics, business development and game user research. His work is carried out in collaboration with companies spanning the industry. He writes about analytics for game development on andersdrachen.com and in trade publications. He is one of the most published experts on game analytics and user profiling. 
Pejman Mirza-Babaei is the User Research Directors at Execution Labs (an incubator for indie developers). He ensures that gameplay experiences meet designers' intent, and he is responsible for UX research and playtesting for projects developed at the Labs. He is also an Assistant Professor at the University of Ontario Institute of Technology, where he researches and teaches game design and development. His main interest is in developing methods to provide formative feedback to indie game developers during the game development cycle to optimize player experiences. Pejman has worked on pre- and post-release evaluation of various titles such as Crysis 2, Split/Second Velocity, Pure, Buzz Quiz World and Sony's Wonderbook, PewDiePai: Legend of Brofist. Pejman is an active member of the IGDA

Games User Research SIG and has presented talks and co-organized workshops at various events. He is currently co-editing a book on games user research.

Andrea Abney is a senior games user researcher at Scopely, where she leads UX research on their current and upcoming slate of games. In her spare time, she conducts lightweight research for Lost Decade Games, an indie game studio based in California. Andrea was previously at Disney Interactive, heading up user research on their Marvel and Star Wars titles. She holds a Master's Degree in Human-Computer Interaction from DePaul University and helped organize the 2014 Games User Research Summit.

Zhu Zhenyu (Cole) is a games user researcher from Tencent Games. He received his Master's degree from TU Eindhoven, focusing on human-technology interaction. Zhu is a regular contributor in combining the psychophysiological approach from UX research within a commercial context. He is also supporting user re- search on League of Legends in China by providing actionable user insights.

\section{Acknowledgements}

We thank the IGDA GUR SIG members and CHI 2012/2013/2014/2015 GUR workshop organizers for their support and encouragement. Lennart thanks NSERC (RGPIN-418622-2012, Discovery Grant) and SSHRC (895-2011-1014, IMMERSe) for their support. Christiane gratefully acknowledges the financial support by the Austrian Federal Ministry of Economy, Family and Youth, and the National Foundation for Research, Technology and Development (Christian Doppler Laboratory for Contextual Interfaces). Pejman thanks his funding from his NSERC Discovery Grant (RGPIN-201405763).

\section{References}

1. Andrea Abney, Brooke White, Jeremy Glick, Andre Bermudez, Paul Breckow, Jason Yow, Rayna Tillinghast-Trickett, and Paul Heath. 2014. Evaluation of recording methods for user test sessions on mobile devices. In Proceedings of the first ACM SIGCHI annual symposium on Computer-human interaction in play (CHI PLAY '14). ACM, New York, NY, USA, 1-8. http://dx.doi.org/10.1145/2658537.2658704

2. ESA, 2015. The 2015 Essential Facts About the Computer and Video Game Industry. http://www.theesa.com/wpcontent/uploads/2015/04/ESA-Essential-Facts2015.pdf

3. Isbister, Katherine, and Noah Schaffer. Game usability: Advancing the player experience. CRC Press, 2008.

4. McGonigal, J. Gaming can make a better world. TED2010 presentation.

https://www.ted.com/talks/jane_mcgonigal_gamin g_can_make_a_better_world?language=en 
5. Pejman Mirza-Babaei, Veronica Zammitto, Jörg Niesenhaus, Mirweis Sangin, and Lennart Nacke. 2013. Games user research: practice, methods, and applications. In CHI '13 Extended Abstracts on Human Factors in Computing Systems (CHI EA '13). ACM, New York, NY, USA, 3219-3222.

http://doi.acm.org/10.1145/2468356.2479651

6. Lennart E. Nacke, Steve Engels, and Pejman MirzaBabaei. 2015. Actionable Inexpensive Games User Research. In Proceedings of the 33rd Annual ACM Conference Extended Abstracts on Human Factors in Computing Systems (CHI EA '15). ACM, New York, NY, USA, 2461-2462.

http://dx.doi.org/10.1145/2702613.2706681

7. Newszoo, 2015. Global Games Market Report 2015 http://www.newzoo.com/product/2015-globalgames-market-report/

8. Superdata 's Monthly Digital Console Report, January 2015. www.superdataresearch.com

9. Magy Seif El-Nasr, Heather Desurvire, Lennart Nacke, Anders Drachen, Licia Calvi, Katherine Isbister, and Regina Bernhaupt. 2012. Game user research. In $\mathrm{CHI}$ '12 Extended Abstracts on Human Factors in Computing Systems (CHI EA '12). ACM, New York, NY, USA, 2679-2682. http://dx.doi.org/10.1145/2212776.2212694.

10. Chek Tien Tan, Pejman Mirza-Babaei, Veronica Zammitto, Alessandro Canossa, Genevieve Conley, and Guenter Wallner. 2015. Tool Design Jam: Designing tools for Games User Research. In Proceedings of the 2015 Annual Symposium on ComputerHuman Interaction in Play (CHI PLAY '15). ACM, New York, NY, USA, 827-831.

http://dx.doi.org/10.1145/2793107.2810263

11. Gareth R. White, Joonhwan Lee, Daniel Johnson, Peta Wyeth, and Pejman Mirza-Babaei. 2015. Crossing Domains: Diverse Perspectives on Players. In Proceedings of the 33rd Annual ACM Conference Extended Abstracts on Human Factors in Compu- ting Systems (CHI EA '15). ACM, New York, NY, USA, 2349-2352.

http://doi.acm.org/10.1145/2702613.2702636

12. Mark, Wilcox. 2014. Mobile Gaming's Dirty Secret, Developer Economics.

http://www.developereconomics.com/mobilegaming-dirty-secret/

13. Veronica Zammitto, Pejman Mirza-Babaei, Ian Livingston, Marina Kobayashi, and Lennart E. Nacke. 2014. Player experience: mixed methods and reporting results. In $\mathrm{CHI}$ '14 Extended Abstracts on Human Factors in Computing Systems (CHI EA '14). ACM, New York, NY, USA, 147-150. http://dx.doi.org/10.1145/2559206.2559239. 